\title{
EXPRESSION OF SIR1-SIR7 GENES IN THE COURSE OF RECURRENT DEPRESSIVE DISORDERS
}

\author{
Edyta Staroń $^{1 *}$, Maria Filip $^{2 *}$, Monika Talarowska ${ }^{3 *}$, Janusz Szemraj $^{4} \&$ Piotr Gałecki $^{2}$ \\ ${ }^{1}$ Świętokrzyskie Psychiatry Centre in Morawica, Branch D, Kielce, Poland \\ ${ }^{2}$ Department of Adult Psychiatry, Medical University of Lodz, Lodz, Poland \\ ${ }^{3}$ University of Lodz, Faculty of Educational Sciences, Institute of Psychology, Department of Personality \\ and Individual Differences, Lodz, Poland \\ ${ }^{4}$ Department of Medical Biochemistry, Medical University of Lodz, Lodz, Poland
}

* Equivalent share of the authors in the compilation of this paper

received: 18.4.2019;

revised: 13.8.2019;

accepted: 30.8 .2019

\section{SUMMARY}

Background: The role of sirtuins as a pathogenetic element of some mental disorders is becoming increasingly more common. They participate in many cellular processes, such as ageing, transcription, apoptosis, inflammatory processes, post-translational modification of proteins, gene transcription silencing, activation of DNA repair mechanisms, and regulation of many metabolic processes. The aim of this paper is to verify the statistical hypothesis assuming the difference in expression at the level of $m R N A$ in genes for sirtuins 1-7 between patients with recurrent depressive disorders ( $r D D)$ and patients from the control group, and the hypothesis assuming the relation between the expression at the level of $m R N A$ for these genes and clinical variables in the course of recurrent depressive disorders.

Subjects and methods: A total of 198 individuals took part in the study (rDD gropup, $N=99$; control group, $N=99$ ).

Results: SIR-1 and SIR-6 expression at the mRNA level was significantly higher among the people with rDD as compared to the subjects from the control group. A reversed relationship was observed for SIR-2, SIR-3, SIR-4 and SIR-5. Statistically significant correlations were observed only in the case of SIR-1 and the number of depression episodes (negative relationship), as well as SIR-5 and the severity of depression measured by the Hamilton Depression Rating Scale (positive relationship).

Conclusions: Expression at the mRNA level for selected sirtuins is a factor that significantly differentiates people with depressive episodes from healthy ones. SIR-1 and SIR-6 expression at the mRNA level was significantly higher among the people with depression as compared to the subjects from the control group. A reversed relationship (also statistically significant) was observed for SIR-2, SIR-3, SIR-4 and SIR-5.

Key words: sirtuins - depression - epigenetics

\section{INTRODUCTION}

In recent years, scientific studies attempting to explain the aetiology of recurrent depressive disorders (RDD) have increasingly referred to the role that epigenetic mechanisms (modification of histones, DNA methylation, or gene expression changes) can play in this phenomenon (Binder 2018, Matosin et al. 2018). These mechanisms, by integrating genetic and environmental factors, play an important role in the inheritance of neurobehavioural patterns and personality traits. This is done by preparing our bodies to cope with the rapidly changing environment. Epigenetic mechanisms, increasing the chances of species survival by significantly shortening the time needed to pass on acquired skills to future generations, in case of environmental conditions that are changing too fast may, paradoxically, cause a lot of damage (Saavedra-Rodríguez \& Feig 2013).

Sir proteins (silent information regulators, sirtuins, SIRT1-SIRT7, SIR1-SIR7) belong to NAD+-dependent deacetylases - enzymes taking part in a catalytic reaction of deacetylation, i.e. splitting the rest of acetic acid from protein substrates (Siedlecka \& Bogusławski 2005, Rowlands et al. 2015). Sirtuins play an important role in the course of many cellular processes, including transcription, apoptosis, inflammatory processes, post-translational protein modification, gene transcription silencing, activation of DNA repair mechanisms, and regulation of intracellular metabolic processes (Kumar et al. 2014, Jęśko et al. 2017), which is why they are involved in the process of ageing and regulating cell life expectancy (Carafa et al. 2016, de Lucia et al. 2017). Their links to cancer (Coppola et al. 2017), neurodegenerative diseases (e.g. Alzheimer's disease, AD) (Scuderi et al. 2014), Huntington's chorea (Quinti et al. 2016), chronic inflammatory diseases, cardiovascular diseases (D'Onofrio et al. 2018) and mental disorders (Alageel et al. 2018) (including bipolar affective disease (Nivoli et al. 2016)) have been demonstrated in recent years. Sirtuins 1, 2 and 3 may also be responsible for the co-occurrence of mental disorders and metabolic diseases (Alageel et al. 2018). The types, locations and effects of individual sirtuins occurring in the human body are presented in Table 1 . 


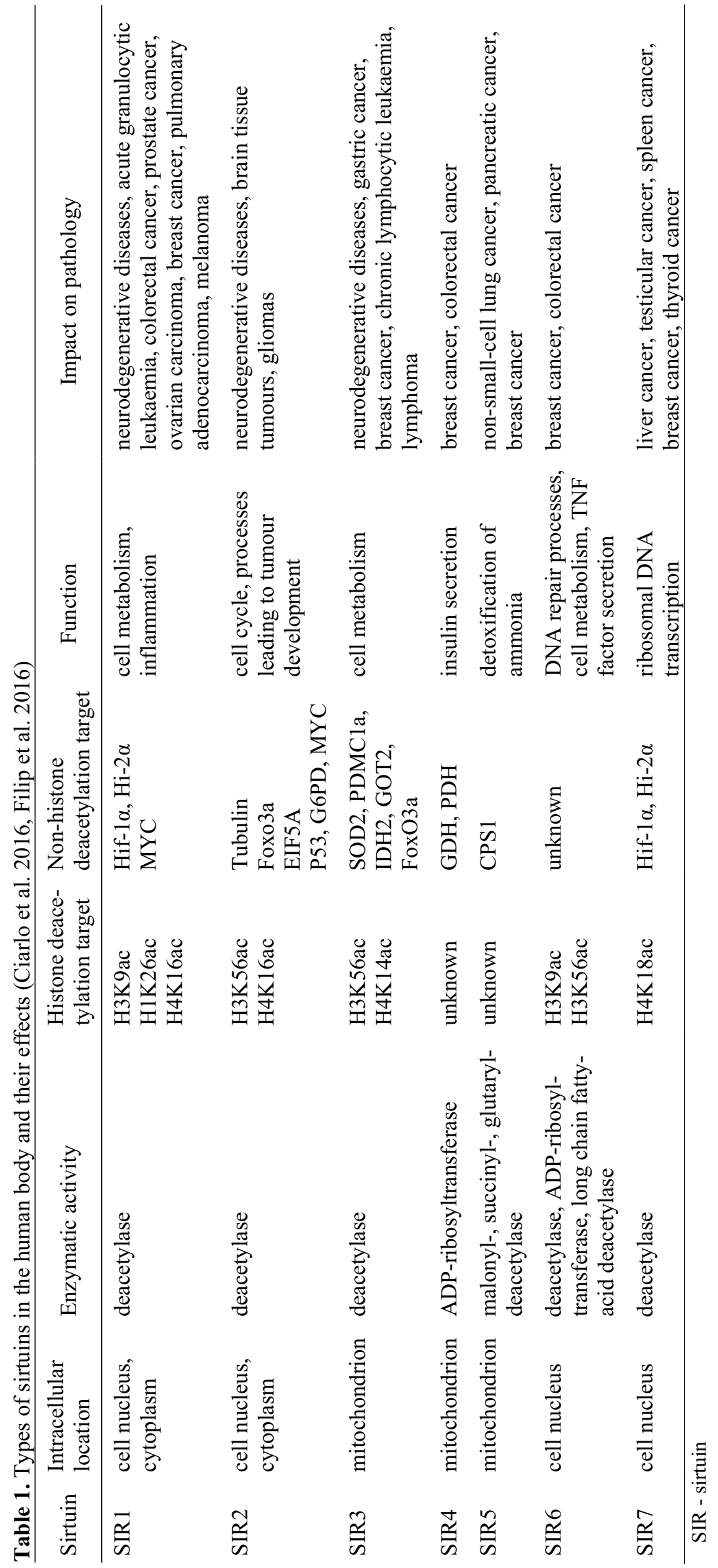


To date, few reports have been published showing the relationship between the expression of sirtuins in patients' bodies and the incidence of mental diseases, including depressive disorders. The aim of this paper is to verify the statistical hypothesis assuming the difference in expression at the mRNA level in genes for sirtuin 1, 2, 3, 4, 5, 6, 7 between patients with recurrent depressive disorders and patients from the control group (i), and the hypothesis assuming the relation between the expression at the level of protein for these genes and clinical variables in the course of recurrent depressive disorders (ii).

\section{SUBJECTS AND METHODS}

\section{Subjects}

A total of 198 individuals took part in the study. The subjects were divided into two groups: group A (experimental group, $\mathrm{N}=99$ ) and group $\mathrm{B}$ (control group, $\mathrm{N}=99$ ). All the subjects were native Poles from central Poland, not related to one another.

The selection of the people for the examined groups was performed at random based on a draw. The respondents made a decision to participate in the study after they had been informed of the purpose and ensured that their participation was voluntary, and the personal details and results of the tests conducted would not be distributed, but used only and exclusively in general comparisons. Each patient gave written consent to participate in the experiment in accordance with the report approved by the Bioethics Committee of the Medical University of Lodz (approval no.: RNN/99/16KE of 19 April 2016).

\section{Group A (experimental)}

The experimental group (recurrent depressive disorders, RDD) consisted of 99 patients hospitalized with the diagnosis of a depressive episode or recurrent depressive disorders at the Department of Adult Psychiatry of the Medical University of Lodz, Poland.

Patients were qualified for the experimental group on the basis of the diagnostic criteria included in ICD10 , i.e. for depressive episode (F32) and recurrent depressive disorders (F33.0-F33.8) (ICD-10 2015). All the patients took part in the study during hospitalization. All patients were taking selective serotonin reuptake inhibitors (SSRIs).

The experimental group included persons hospitalized psychiatrically for the first time and not treated earlier due to depressive disorders, as well as persons treated pharmacologically for many years, admitted to the unit in order to modify their therapy or due to deterioration of their health condition (another affective episode). The exclusion criterion was the presence of axis I and II disorders, other than depressive episodes, as well as chronic somatic diseases. Other exclusion criteria included cancer, major neurological diseases and severe and chronic inflammatory diseases. In all cases, data on the course of the disease were collected using the Composite International Diagnostic Interview (CIDI) (Kessler \& Ustün 2004). Medical data on the course of the disease were obtained directly from patients, from attending physicians and from medical records (with patients' consent).

\section{Group B (control)}

The control group (CG) consisted of 99 healthy people with negative family history of mental diseases. The assessment of mental health in this case was also based on CIDI criteria (Kessler \& Ustün 2004). People with axis I and axis II disorders were excluded from participation in the study. As in the experimental group, the exclusion criteria were neurological, neoplastic and inflammatory diseases.

\section{Methods \\ Assessment of intensification of recurrent depressive disorder symptoms}

The Hamilton Depression Rating Scale (HDRS, HAM-D) was used to evaluate the dynamics of recurrent depressive disorder symptoms intensification (Hamilton 1960). A scoring system developed by Demyttenaere and De Fruyt (2003) was applied when analysing the intensity of depressive episode symptoms. Furthermore, the CIDI questionnaire (version 3.0) was used in the experiment. This tool is based on the diagnostic criteria of the ICD-10 and DSM-V classifications, and is recommended by WHO and WMH for epidemiological studies in psychiatry. It enables the estimation of mental disorders dissemination and the evaluation of their advancement and subjective burden of the disease (Kessler \& Ustün 2004).

In each case, an evaluation of the mental state and assessment of the severity of depressive disorders were conducted by the same person, i.e. a psychiatrist. The CIDI questionnaire was used at the stage of patients qualification to participate in the study. The examination with the use of the HDRS scale was performed on the day of inclusion in the experiment.

\section{Genetic analysis}

Total RNA isolation

Total RNA isolation from the patients' blood samples (leukocyte monolayer cells) using a RNA extraction reagent, TRIZOL (Invitrogen Life Technologies), according to the standard acid-guanidinium-phenolchlorophorm method was performed usining modified Chomczyński metod.

\section{Quality analysis of isolated $R N A$}

The quality of total RNA was checked with Agilent RNA 6000 Nano Kit (Agilent Technologies) in accordance with the manufacturer's recommendations. The quality of isolated RNA was checked using 2100 Bioanalyzer (Agilent Technologies). The level of degradation of total RNA was determined with the use of an electrophoretogram and RIN values recorded. Only the samples with RIN value $>7$ were subject to further analysis. 


\section{RT-PCR reverse transcription}

An RT reaction was carried out using TaqMan ${ }^{\circledR}$ RNA Reverse Transcription Kit (Applied Biosystems) based on the manufacturer's recommendations, using specific delivered by Applied Biosystems.

\section{Real-Time PCR reaction}

Real-Time PCR reaction was conducted using TaqMan ${ }^{\circledR}$ Universal PCR Master Mix, No UNG (Applied Biosystems) according to the protocol provided by the manufacturer.

Each target probe was amplified in a separate 96well plate. All samples were incubated at $50^{\circ} \mathrm{C}$ for 2 minutes and at $95^{\circ} \mathrm{C}$ for 10 minutes, and then cycled at $95^{\circ} \mathrm{C}$ for 30 seconds, at $60^{\circ} \mathrm{C}$ for 30 seconds and at $72^{\circ} \mathrm{C}$ for 1 minute; 40 cycles were performed in total. Fluorescence emission data were captured and mRNA levels were quantified using the critical threshold $(\mathrm{Ct})$ value. Analyses were performed with ABI Prism 7000 (SDS Software). Controls without RT and with no template cDNA were performed with each assay. Relative gene expression levels were obtained using the $\Delta \Delta \mathrm{Ct}$ standard 2- $\Delta \Delta \mathrm{ct}$ calculations and expressed as a fold change of the control sample. Amplification specific transcripts were further confirmed by obtaining melting curve profiles.

\section{Statistical analysis of results}

Selected methods of descriptive statistics and methods of statistical reasoning were used in the statistical analysis of the collected material. During statistical verification of the hypotheses, a two-tailed critical area was assumed.
Appropriate structural indicators, i.e. prevalence of a given trait expressed in percentage terms, were applied in the description of qualitative features in the examined group of affected patients and the control group. An arithmetic mean (M) was calculated to describe the value of average quantitative features. The scope of values (with the minimum and maximum value determined) as well as standard deviation (SD) were assumed as measures of dispersion.

The nature of the distribution of all variables was examined with the Shapiro-Wilk test. The following non-parametric tests were applied with reference to non-parametric variables for statistical comparisons between the examined groups: Pearson's chi-squared test (for qualitative variables) and Mann-Whitney U test in the case of two independent groups (for quantitative variables). Spearman's rank correlation coefficient was used to evaluate the correlations between the analysed variables. The materiality level for all the statistical methods applied was set at $\mathrm{p}<0.05$ (Kirkwood \& Sterne 2003). All statistical calculations were conducted using computer software STATISTICA PL, version 13.1.

\section{RESULTS}

The social and demographic characteristics of the studied groups and the information regarding the course of the underlying disease, significant from the perspective of the experiment, are presented in Table 2.

Statistically significant differences between the examined groups in terms of $\operatorname{sex}\left(\chi^{2}=4.254, p=0.039\right)$ and age of the subjects (in each age group) were observed.

Table 2. The characteristics of the studied groups in terms of demographic features $(\mathrm{N}=198)$ and the course of the disease $(\mathrm{N}=99)$

\begin{tabular}{|c|c|c|c|c|c|c|}
\hline \multirow[t]{2}{*}{ Women/men - n (\%) } & \multicolumn{2}{|c|}{$\begin{array}{c}\mathrm{N}=198 \\
143 / 55(72.22 / 27.78)\end{array}$} & \multicolumn{2}{|c|}{$\begin{array}{c}\text { Group A; } N=99 \\
78 / 21(78.79 / 21.21)\end{array}$} & \multicolumn{2}{|c|}{$\begin{array}{c}\text { Group B; N=99 } \\
65 / 34(65.66 / 34.34)\end{array}$} \\
\hline & $\mathrm{N}$ & $\%$ & $\mathrm{~N}$ & $\%$ & $\mathrm{~N}$ & $\%$ \\
\hline \multicolumn{7}{|l|}{ Age } \\
\hline$<20$ years & 7 & 3.54 & 1 & 1.01 & 6 & 6.06 \\
\hline $21-35$ years & 87 & 43.94 & 9 & 9.09 & 78 & 78.79 \\
\hline $36-50$ years & 34 & 17.17 & 21 & 21.21 & 13 & 13.13 \\
\hline $51-60$ years & 33 & 16.67 & 31 & 31.31 & 2 & 2.02 \\
\hline$>60$ & 37 & 18.69 & 37 & 37.37 & 0 & 0.00 \\
\hline
\end{tabular}

Group A $(\mathrm{N}=99)$

HDRS - M (SD)

Number of depression episodes; $\mathrm{M}$ (SD)

$\mathrm{M}=24.39(7.08)$

$\mathrm{M}=3.33(1.69)$

Duration of the disease - N (\%)

$<20$ years

21-35 years

$32(32.32)$

36-50 years

$34(34.34)$

$51-60$ years

$19(19.19)$

$>60$ years

$12(12.12)$

HDRS - Hamilton Depression Rating Scale on the day of inclusion in the study; Group A - experimental group;

Group B - control group; $\mathrm{M}$ - mean; $\mathrm{SD}$ - standard deviation 
Table 3. Mean values, standard deviation, minimum and maximum values of analysed variables in the examined $\underline{\operatorname{group}(\mathrm{N}=198)}$

\begin{tabular}{lccc}
\hline $\begin{array}{l}\text { mRNA } \\
(2-\Delta \Delta \mathrm{ct})\end{array}$ & M (SD) & Min. & Max. \\
\hline SIR-1 & $0.330(0.299)$ & 0.021 & 0.938 \\
SIR-2 & $0.200(0.162)$ & 0.022 & 0.603 \\
SIR-3 & $0.136(0.090)$ & 0.019 & 0.343 \\
SIR-4 & $0.126(0.090)$ & 0.011 & 0.337 \\
SIR-5 & $0.125(0.083)$ & 0.013 & 0.301 \\
SIR-6 & $0.213(0.153)$ & 0.030 & 0.514 \\
SIR-7 & $0.121(0.079)$ & 0.011 & 0.367 \\
\hline
\end{tabular}

SIR-1 - sirtuin 1; SIR-2 - sirtuin 2; SIR-3 - sirtuin 3; SIR-4 - sirtuin 4; SIR-5 - sirtuin 5; SIR-6 - sirtuin 6; SIR-7 - sirtuin 7; M - mean; SD - standard deviation; Min. - minimum value; Max. - maximum value

Table 3 presents mean values, standard deviation, minimum and maximum values of concentration and expression on mRNA level for sirtuins 1, 2, 3, 4, 5, 6 and 7 in the studied group $(\mathrm{N}=198)$. Table 3 includes a comparison of the analysed variables in patients with recurrent depressive disorders $(\mathrm{N}=99)$ and healthy individuals $(\mathrm{N}=99)$.

Statistically significant differences were observed between the subjects with recurrent depressive disorders and the control group in terms of the analysed variables (Table 4). SIR-1 and SIR-6 expression at the mRNA level was significantly higher among the people with RDD as compared to the subjects from the control group. A reversed relationship (also statistically significant) was observed for SIR-2, SIR-3, SIR-4 and SIR-5.

\section{Correlations}

Table 5 presents the results of a correlation analysis between expression at the mRNA level for the analysed compounds and clinical exponents of recurrent depressive disorders.

Statistically significant correlations were observed only in the case of SIR-1 and the number of depression episodes (negative relationship), as well as SIR-5 and the severity of depression measured by the Hamilton Depression Rating Scale (positive relationship).

Table 4. Comparison of analysed variables in patients with recurrent depressive disorders ( $\mathrm{N}=99)$ and healthy individuals $(\mathrm{N}=99)$

\begin{tabular}{|c|c|c|c|c|c|c|c|c|}
\hline \multirow{2}{*}{$\begin{array}{l}\text { mRNA } \\
(2-\Delta \Delta c t)\end{array}$} & \multicolumn{3}{|c|}{ Group A } & \multicolumn{3}{|c|}{ Group B } & \multicolumn{2}{|c|}{ Mann-Whitney U test } \\
\hline & $\mathrm{M}(\mathrm{SD})$ & Min. & Max. & $\mathrm{M}(\mathrm{SD})$ & Min. & Max. & Z & $\mathrm{p}$ \\
\hline SIR-1 & $0.062(0.021)$ & 0.021 & 0.118 & $0.599(0.138)$ & 0.103 & 0.938 & -12.1 & $<0.01^{*}$ \\
\hline SIR-2 & $0.337(0.112)$ & 0.152 & 0.603 & $0.062(0.044)$ & 0.022 & 0.370 & 11.9 & $<0.01^{*}$ \\
\hline SIR-3 & $0.206(0.064)$ & 0.096 & 0.343 & $0.066(0.048)$ & 0.019 & 0.272 & 11.1 & $<0.01^{*}$ \\
\hline SIR-4 & $0.206(0.056)$ & 0.084 & 0.337 & $0.047(0.022)$ & 0.011 & 0.124 & 12.1 & $<0.01 *$ \\
\hline SIR-5 & $0.200(0.047)$ & 0.067 & 0.301 & $0.049(0.018)$ & 0.013 & 0.096 & 12.1 & $<0.01^{*}$ \\
\hline SIR-6 & $0.073(0.022)$ & 0.034 & 0.129 & $0.353(0.085)$ & 0.030 & 0.514 & -11.9 & $<0.01^{*}$ \\
\hline SIR-7 & $0.190(0.051)$ & 0.087 & 0.367 & $0.053(0.022)$ & 0.011 & 0.121 & 12.1 & $<0.01^{*}$ \\
\hline
\end{tabular}

SIR-1 - sirtuin 1; SIR-2 - sirtuin 2; SIR-3 - sirtuin 3; SIR-4 - sirtuin 4; SIR-5 - sirtuin 5; SIR-6 - sirtuin 6; SIR-7 - sirtuin 7; Group A - experimental group; Group B - control group; $\mathrm{M}$ - mean; $\mathrm{SD}$ - standard deviation; Min. - minimum value; Max. - maximum value; ${ }^{*}-$ p statistically significant, $<0.01$

Table 5. Correlation between clinical exponents of depressive disorders and analysed variables $(\mathrm{N}=99)$

\begin{tabular}{|c|c|c|c|}
\hline Variable & & R Spearman & $\mathrm{p}$ \\
\hline SIR-1 mRNA $(2-\Delta \Delta c t)$ & number of depression episodes & -0.198 & $0.049^{*}$ \\
\hline$\&$ & HDRS & -0.168 & 0.097 \\
\hline SIR-2 mRNA $(2-\Delta \Delta c t)$ & number of depression episodes & 0.080 & 0.431 \\
\hline$\&$ & HDRS & -0.085 & 0.404 \\
\hline SIR-3 mRNA $(2-\Delta \Delta c t)$ & number of depression episodes & 0.036 & 0.724 \\
\hline$\&$ & HDRS & -0.068 & 0.504 \\
\hline SIR-4 mRNA $(2-\Delta \Delta c t)$ & number of depression episodes & -0.126 & 0.215 \\
\hline$\&$ & HDRS & -0.023 & 0.820 \\
\hline SIR-5 mRNA $(2-\Delta \Delta c t)$ & number of depression episodes & 0.033 & 0.746 \\
\hline$\&$ & HDRS & 0.214 & $0.033^{*}$ \\
\hline SIR-6 mRNA $(2-\Delta \Delta c t)$ & number of depression episodes & -0.108 & 0.287 \\
\hline$\&$ & HDRS & 0.129 & 0.204 \\
\hline SIR-7 mRNA (2- $\Delta \Delta c t)$ & number of depression episodes & -0.036 & 0.723 \\
\hline$\&$ & HDRS & -0.033 & 0.744 \\
\hline
\end{tabular}

SIR-1 - sirtuin 1; SIR-2 - sirtuin 2; SIR-3 - sirtuin 3; SIR-4 - sirtuin 4; SIR-5 - sirtuin 5; SIR-6 - sirtuin 6; SIR-7 - sirtuin 7; HDRS I - Hamilton Depression Rating Scale on the day of inclusion in the study; * p - statistically significant, $<0.05$ 


\section{DISCUSSION}

This paper is one of the few publications (compare Abe et al. (2011)) analysing the expression of selected sirtuins among people with depressive episodes, and the only one we found presenting this issue in relation to the Polish population. The statistical hypotheses formulated by us were partially confirmed in the conducted research. The mRNA level for SIR-1 and SIR-6 was statistically significantly higher in the control group. In the case of SIR-2, SIR-3, SIR-4, SIR-5 and SIR-7 a significantly higher concentration at the mRNA level was observed in the group of patients with RDD (hypothesis one). The frequency of relapses was conducive to the reduction of SIR- 1 at the mRNA level, and the intensification of disease symptoms measured by the HDRS scale was accompanied by an increase in SIR-5 concentration at the mRNA level (hypothesis two).

Results consistent with those obtained by us can be found in literature. In an experiment conducted by Lee et al. (2018), SIR3 expression at both mRNA and protein levels was significantly lower in patients with Alzheimer's disease (SIR3 prevents, among others, mitochondrial dysfunctions induced by $\mathrm{p} 53$ and neuronal damage). Also SIR 1, mainly responsible for neuroprotection, and SIR 2, conducive to neurodegeneration, can play a role in the occurrence of $\mathrm{AD}$ symptoms (Scuderi et al. 2014). Sirtuin 1 is particularly important in this group of patients, where it has been shown that it significantly reduces the rate of neurodegenerative changes occurring in the hippocampus region (Kim et al. 2007). Interesting research findings were presented by Nivoli et al. (2016). In the case of the rs 10997870 single nucleotide polymorphism (SNP), the GG homozygote for the SIR1 gene was associated with the intensity of psychomotor agitation, anxiety, depression symptoms and the risk of committing suicide among patients with bipolar affective disease $(\mathrm{N}=180)$. In $\mathrm{AD}$ patients, this correlation was observed between the severity of depressive symptoms and SNP rs10410544 for SIR2 (Porcelli et al. 2013). Moreover, the level of SIR1 expression positively correlates with the efficiency of episodic memory, the speed and efficiency of information processing, and the global level of cognitive performance (Mahady et al. 2018). Interestingly, sirtuin 1 and 7 level modulation mediates the reduction of the level of experienced anxiety (Reddy et al. 2016).

The exact mechanism of the influence of individual sirtuins on the occurrence of mental disorders and neurodegenerative diseases has not yet been clarified (Alageel et al. 2018). According to Mendes et al. (2017) and Bortell et al. (2018), sirtuins 1, 2, 6 and 7 may be of key importance for the regulation of inflammatory response. SIRT1 has been shown to suppress the activity of NF- $\mathrm{kb}$, the main regulator of inflammatory cell response. It reduces the production of COX-2 and iNOS and increases the expression of the antioxidant gene, which is also responsible for suppressing inflammation. SIR2 activity involves deacetylation of $\mathrm{p} 65 \mathrm{NF}-\kappa \beta$ subunit and RIP-1, while SIRT6 interacts with $\mathrm{p} 65 / \mathrm{RelA}$, directly related to the NF- $\kappa \beta$ promoter region, and suppresses transcriptional activity. Moreover, recent studies have shown that the lack of SIRT7 causes an increase in inflammation, which shows that SIRT7 reduces inflammation (Mendes et al. 2017, Bortell et al. 2018).

Ferland et al. (2013) emphasise that sirtuin activity contributes to molecular cascade changes and histone acetylation within the hippocampus in response to chronic stress stimuli. On the other hand, sirtuin 5 increases permeability of the blood-brain barrier (BBB) (Diaz-Cañestro et al. 2018), which may be crucial for the activation of neuroinflammatory processes. SIR2 deficit (as opposed to sirtuin 3) increases bacterial phagocytosis by macrophages, reducing the risk of infection (Ciarlo et al. 2018). Furthermore, according to Erburu et al. (2017), SIR2 inhibition has an antidepressant effect (stimulation of neuroplastic processes) through its effect on glutamatergic and serotonergic systems in the area of the prefrontal cortex. Similar results were obtained in the research conducted by Muñoz-Cobo et al. (2017). On the other hand, SIR7 (Burg et al. 2018) has a protective effect on the immune system and additionally stimulates neurogenesis (Burg et al. 2018). The role of sirtuins in neuroplasticity processes (Abe-Higuchi et al. 2016) and in neurogenesis is probable, especially in the case of sirtuin 6 (Okun et al. 2017). Abe-Higuchi et al. (2016) demonstrated that chronic stress reduces SIR1 expression in the dentate gyrus of the hippocampus, which increases the probability of occurrence of depression-like behaviour. Hippocampal activation of SIR1 increases the phosphorylation level of extracellular protein kinases regulated by signal 1 and 2 under stressful conditions, while virus activation and inhibition of hippocampal extracellular protein regulation of kinase 2 leads to antidepressant and prodepressant behaviour (Abe-Higuchi et al. 2016).

\section{Limitations}

- All patients taking part in the study were taking SSRIs (selective serotonin reuptake inhibitors). Be aware of the possible effects of pharmacotherapy on gene expression.

- The limitation of the study may also be the fact that we used peripheral blood, not cerebrospinal fluid. For this reason, other factors beyond the mental state could also have an effect on gene expression.

\section{CONCLUSIONS}

Expression at the mRNA level for selected sirtuins is a factor that significantly differentiates people with depressive episodes from healthy ones. SIR-1 and SIR-6 
expression at the mRNA level was significantly higher among the people with depression as compared to the subjects from the control group. A reversed relationship (also statistically significant) was observed for SIR-2, SIR-3, SIR-4 and SIR-5.

\section{Acknowledgements:}

The research was financed with grants for scientific research awarded by the Medical University of Lodz No. 503/5-062-02/503-51-010-18 and No. 502-03/5062-02/502-54-216.

\section{Conflict of interest: None to declare.}

\section{Contribution of individual authors:}

Edyta Staroń - study design, data collection, data interpretation, preparation of manuscript.

Maria Filip - study design, data collection, data interpretation, preparation of manuscript, literature analysis.

Monika Talarowska - study design, data interpretation, preparation of manuscript, literature analysis.

Janusz Szemraj - data interpretation.

Piotr Gałecki - Study design, data interpretation, preparation of manuscript, literature analysis, funds collection.

\section{References}

1. Abe N, Uchida S, Otsuki K, Hobara T, Yamagata H, Higuchi $F$ et al: Altered sirtuin deacetylase gene expression in patients with a mood disorder. $J$ Psychiatr Res 2011; 45:1106-12

2. Abe-Higuchi $N$, Uchida S, Yamagata H, Higuchi $F$, Hobara T, Hara $K$ et al: Hippocampal sirtuin 1 signaling mediates depression-like behavior. Biol Psychiatry 2016; 80:815-26

3. Alageel A, Tomasi J, Tersigni C, Brietzke E, Zuckerman $H$, Subramaniapillai $M$ et al: Evidence supporting a mechanistic role of sirtuins in mood and metabolic disorders. Prog Neuropsychopharmacol Biol Psychiatry 2018; 86:95-101

4. Binder EB: Dissecting the molecular mechanisms of gene $x$ environment interactions: implications for diagnosis and treatment of stress-related psychiatric disorders. Eur $J$ Psychotraumatol 2018; 8(suppl 5):1412745

5. Bortell N, Basova L, Najera JA, Morsey B, Fox HS \& Marcondes MCG: Sirtuin 1-chromatin-binding dynamics points to a common mechanism regulating inflammatory targets in SIV infection and in the aging brain. $J$ Neuroimmune Pharmacol 2018; 13:163-78

6. Burg N, Bittner $S$ \& Ellwardt E: Role of the epigenetic factor Sirt7 in neuroinflammation and neurogenesis. Neurosci Res 2018; 131:1-9

7. Carafa V, Rotili D, Forgione M, Cuomo F, Serretiello E, Hailu GS et al: Sirtuin functions and modulation: from chemistry to the clinic. Clin Epigenetics 2016; 8:61

8. Ciarlo E, Heinonen T, Lugrin J, Acha-Orbea H, Le Roy D, Auwerx J: Sirtuin 3 deficiency does not alter host defenses against bacterial and fungal infections. Sci Rep 2017; 7:3853
9. Ciarlo E, Heinonen T, Théroude C, Herderschee J, Mombelli M, Lugrin J: Sirtuin 2 deficiency increases bacterial phagocytosis by macrophages and protects from chronic staphylococcal infection. Front Immunol 2017; 8:1037

10. Coppola N, de Stefano G, Panella M, Onorato L, Iodice V, Minichini $C$ et al: Lowered expression of microRNA-125a$5 p$ in human hepatocellular carcinoma and up-regulation of its oncogenic targets sirtuin-7, matrix metalloproteinase11, and c-Raf. Oncotarget 2017; 8:25289-99

11. de Lucia C, Murphy $T$ \& Thuret S: Emerging molecular pathways governing dietary regulation of neural stem cells during aging. Front Physiol 2017; 8:17

12. Demyttenaere $K \&$ De Fruyt J: Getting what you ask for: on the selectivity of depression rating scales. Psychothery Psychosom 2003; 72:61-70

13. Diaz-Cañestro C, Merlini M, Bonetti NR, Liberale L, Wüst $P$, Briand-Schumacher $S$ : Sirtuin 5 as a novel target to blunt blood-brain barrier damage induced by cerebral ischemia/reperfusion injury. Int J Cardiol 2018; 260:148-55

14. D'Onofrio N, Servillo L \& Balestrieri ML: SIRT1 and SIRT6 signaling pathways in cardiovascular disease protection. Antioxid Redox Signal 2018; 28:711-32

15. Erburu M, Muñoz-Cobo I, Diaz-Perdigon T, Mellini P, Suzuki T, Puerta E et al: SIRT2 inhibition modulate glutamate and serotonin systems in the prefrontal cortex and induces antidepressant-like action. Neuropharmacology 2017; 117:195-208

16. Ferland CL, Hawley WR, Puckett RE, Wineberg K, Lubin $F D$, Dohanich GP et al: Sirtuin activity in dentate gyrus contributes to chronic stress-induced behavior and extracellular signal-regulated protein kinases 1 and 2 cascade changes in the hippocampus. Biol Psychiatry 2013; 74:927-35

17. Filip M, Kuśmierek M, Bobińska $K \&$ Galecki P. The role of sirtuins in selected mental disorders. Pharmacotherapy in Psychiatry and Neurology 2016; 32:33-9

18. Hamilton M: A rating scale for depression. J Neurol Neurosurg Psychiatry 1960; 23:56-62

19. International Statistical Classification of Diseases and Related Health Problems 10th Revision (ICD-10). World Health Organization, Genewa, 2015.

20. Jeśko H, Wencel P, Strosznajder RP \& Strosznajder JB: Sirtuins and Their Roles in Brain Aging and Neurodegenerative Disorders. Neurochem Res 2017; 42:876-90

21. Kessler RC \& Ustün TB: The World Mental Health (WMH) Survey Initiative Version of the World Health Organization (WHO) Composite International Diagnostic Interview (CIDI). Int J Methods Psychiatr Res 2004; 13:93-121

22. Kim D, Nguyen MD, Dobbin MM, Fischer A, Sananbenesi $F$, Rodgers JT et al: SIRT1 deacetylase protects against neurodegeneration in models for Alzheimer's disease and amyotrophic lateral sclerosis. EMBO J 2007; 26:3169-79

23. Kirkwood B \& Sterne J: Essential medical statistics, 2nd edition. Wiley-Bleckwell, 2003

24. Kumar R, Mohan N, Upadhyay AD, Singh AP, Sahu V, Dwivedi $S$ et al: Identification of serum sirtuins as novel noninvasive protein markers for frailty. Aging Cell 2014; 13:975-80

25. Lee J, Kim Y, Liu T, Hwang YJ, Hyeon SJ, Im $H$ et al: SIRT3 deregulation is linked to mitochondrial dysfunction in Alzheimer's disease. Aging Cell 2018; 17

26. Mahady L, Nadeem M, Malek-Ahmadi M, Chen K, Perez $S E \&$ Mufson EJ: Frontal Cortex Epigenetic Dysregu- 
lation During the Progression of Alzheimer's Disease. J Alzheimers Dis 2018; 62:115-31

27. Matosin N, Halldorsdottir $T$ \& Binder EB: Understanding the molecular mechanisms underpinning gene by environment interactions in psychiatric disorders: the FKBP5 model. Biol Psychiatry 2018; 83:821-30

28. Mendes KL, Lelis DF \& Santos SHS: Nuclear sirtuins and inflammatory signaling pathways. Cytokine Growth Factor Rev 2017; 38:98-105

29. Muñoz-Cobo I, Belloch FB, Díaz-Perdigón T, Puerta E \& Tordera RM: SIRT2 inhibition reverses anhedonia in the VGLUT1+/- depression model. Behav Brain Res 2017; 335:128-31

30. Nivoli A, Porcelli S, Albani D, Forloni G, Fusco F, Colom $F$ et al: Association between Sirtuin 1 Gene rs 10997870 Polymorphism and Suicide Behaviors in Bipolar Disorder. Neuropsychobiology 2016; 74:1-7

31. Okun E, Marton D, Cohen D, Griffioen K, Kanfi Y, Illouz $T$ et al: Sirt6 alters adult hippocampal neurogenesis. PLoS One 2017; 12:e0179681

32. Porcelli S, Salfi R, Politis A, Atti AR, Albani D, Chierchia $A$ et al: Association between Sirtuin 2 gene rs10410544 polymorphism and depression in Alzheimer's disease in two independent European samples. $J$ Neural Transm (Vienna) 2013; 120:1709-15
33. Quinti L, Casale M, Moniot S, Pais TF, Van Kanegan MJ, Kaltenbach LS et al: SIRT2- and NRF2-Targeting Thiazole-Containing Compound with Therapeutic Activity in Huntington's Disease Models. Cell Chem Biol 2016; 23:849-61

34. Reddy BR, Maitra S, Jhelum P, Kumar KP, Bagul PK, Kaur $G$ et al: Sirtuin 1 and 7 mediate resveratrol-induced recovery from hyper-anxiety in high-fructose-fed prediabetic rats. J Biosci 2016; 41:407-17

35. Rowlands BD, Lau CL, Ryall JG, Thomas DS, Klugmann $M$, Beart $P M$ et al: Silent information regulator 1 modulator resveratrol increases brain lactate production and inhibits mitochondrial metabolism, whereas SRT1720 increases oxidative metabolism. J Neurosci Res 2015; 93:1147-56

36. Saavedra-Rodríguez L \& Feig LA: Chronic social instability induces anxiety and defective social interactions across generations. Biol Psychiatry 2013; 73:44-53

37. Scuderi C, Stecca C, Bronzuoli MR, Rotili D, Valente $S$ \& Mai A: Sirtuin modulators control reactive gliosis in an in vitro model of Alzheimer's disease. Front Pharmacol 2014; 5:89

38. Siedlecka K \& Bogustawski W: Sirtuins - longevity enzymes? Polish Gerontology 2005; 3:147-52

Correspondence:

Prof. Monika Talarowska, MD

Klinika Psychiatrii Dorostych, Uniwersytet Medyczny w Łodzi ul. Aleksandrowska 159, 91-229 Łódź, Poland

E-mail: talarowskamonika@wp.pl 